\title{
Relationships and implications for complementary and alternative medicine in Aotearoa New Zealand: A discussion paper
}

\section{Ngā hononga me ngā putanga ake mō te rongoā tāpiri, kaupapa tuarua hoki i Aotearoa: He puka kōrerorero}

Maria Te Huia RN, PGCertTT, MHealSc, Senior Academic, Centre for Health \& Social Practice, Wintec, Hamilton, NZ

Christine Mercer RN, PhD, Independent Professional Supervisor, Tauranga, \& Academic Staff Member, Wintec, Hamilton, NZ

\section{Abstract}

The purpose of this paper is to initiate a discussion on contextualising the relationship between the nursing profession and complementary and alternative medicine (CAM) within Aotearoa New Zealand. There is limited research and data linking complementary and alternative medicine to nursing or how this could be integrated into health care delivery. The authors' intentions are to raise awareness of a trend within health and wellness that could have implications for the nursing profession in Aotearoa New Zealand. Existing knowledge from overseas research is discussed to raise awareness on complementary and alternative medicine knowledge and any perceptions or educational needs nurses may require when considering the utilisation of complementary and alternative medicine. A range of questions are presented aimed at highlighting areas of development and future research for nursing in Aotearoa New Zealand if complementary and alternative medicine therapies or theory are applied within mainstream health care settings.
\end{abstract}

\section{Keywords / Ngā kupu matua}

complementary and alternative medicine / ngā rongoā tāpiri, kaupapa tuarua hoki; cultural safety / haumaru ahurea; integrative medicine / ngā rongoā tūhono; nursing / te mahi tapuhi; New Zealand / Aotearoa

Te Huia, M., \& Mercer, C. (2019). Relationships and implications for complementary and alternative medicine in Aotearoa New Zealand: A discussion paper. Nursing Praxis in New Zealand, 35(3), 26-33. 


\section{Nursing Praxis in New Zealand}

\section{Introduction}

The use of complementary and alternative medicines (CAM) and therapies have increased in the past 20 years (Frass et al., 2012) as reflected in the literature (National Center for Complementary and Integrative Health [NCCIH], 2016a; World Health Organization [WHO], 2013). Data from Europe showed that CAM use in the United Kingdom was between 6\% - 71\%, Germany $4.6 \%$ - 62\%, Turkey $48 \%$ - 86\%, Switzerland 5\% - $57 \%$, Sweden 5\% - 64\%, Norway 9\% - 53\%, Denmark 45\% - 59\%, Italy $16 \%$ - $84 \%$, Finland $11 \%-43 \%$, and Spain 15\% - 47\% (Eardley et al., 2010) whereas in America $38 \%$ of adults and $12 \%$ of children had used some form of CAM (NCClH, 2016a). The global trend of increased CAM use has also influenced health approaches and practices in Aotearoa New Zealand with a higher rate of consumer demands for a holistic approach to health needs and increased use of CAM (Ministerial Advisory Committee on Complementary and Alternative Health [MACCAH], 2002; 2004). The purpose of this paper is to explore three factors that emerge from the works related to CAM. Firstly, there is a need for clarification of the use and definition of CAM within the context of mainstream health services, and what the use and/ or integration of CAM might mean for the practice of health practitioners pertaining to safety and policy (NCCIH, 2016b; Nicholson, 2006). Secondly, due to the complexity of CAM practices and therapies, this paper looks at generating a clearer picture on the relationship nursing has with CAM in the current Aotearoa New Zealand health profile. Finally, it is proposed here that it would be useful to engage in a more detailed investigation and scholarly discussion around how Aotearoa New Zealand nurses, as a profession, might be affected by this trend. Coupled with this discussion is consideration of the use of CAM therapies in mainstream and rongoā Māori (traditional Māori healing practices) within the context of providing culturally safe practice (Hilbers \& Lewis, 2013; Mark \& Chamberlain, 2012).
Currently there is limited Aotearoa New Zealand-based research on the use of CAM in mainstream (Western conventional or orthodox medicine) contexts. For this reason, papers and research from within New Zealand older than 2008 have been included as these were considered relevant and essential to formulate future direction on the use of CAM in New Zealand.

\section{Background}

The term CAM includes many facets, definitions, and therapeutic approaches as influenced by professional position and/or cultural context (Baer, 2016). A seminal definition of CAM, developed by a panel at the United States' Office for Alternative Medicine of the National Institutes of Health conference in 1997, is:

\begin{abstract}
Complementary and alternative medicine (CAM) is a broad domain of healing resources that encompasses all health systems, modalities, and practices, and their accompanying theories and beliefs, other than those intrinsic to the politically dominant health system of a particular society or culture in a given historical period. CAM includes all such practices and ideas self-identified by their users as preventing or treating illness or promoting health and wellbeing. (O'Connor et al, 1997, p. 50).
\end{abstract}

This definition was adopted by the National Center for Complementary and Integrative Health ( $\mathrm{NCClH}$ ) and underpinned the Aotearoa New Zealand MACCAH reports (2002; 2004) prepared for the Minister of Health. A more contemporary definition used by the CAMbrella Project in Europe includes a holistic experiential approach:

CAM, as utilised by European citizens, represents a variety of different medical systems and therapies based on the knowledge, skills and practices derived from theories, philosophies and experiences used to maintain and improve health, as well as to prevent, diagnose, relieve or treat physical and mental illnesses. CAM therapies are mainly used outside conventional health care, but in many countries some therapies 


\section{Nursing Praxis in New Zealand}

are being adopted or adapted by conventional health care. (EUROCAM, 2014, p. 10).

\section{Implications of complementary and alternative medicine}

The generic use of the term CAM is problematic (Gale, 2014) and can lead to misperceptions and/or misunderstandings for the consumer and health care provider. For this paper the definitions from MACCAH (2002) and the NCCIH (2016a) will be used to position CAM as an adjunct therapy, where treatment is used to complement mainstream interventions for a consumer in contrast to an alternative approach (Tabish, 2008). The term alternative is applied when therapies are used instead of mainstream treatments. Furthermore, the term traditional healing methods is used when "referring to the knowledge, skills, and practices based on the theories, beliefs, and experiences of indigenous cultures" (WHO, 2013, p. 15). Ahuriri-Driscoll, Barker, Hepi and Hudson (2009) emphasised the relevance of Indigenous approaches to health care within Aotearoa New Zealand that acknowledged rongoā Māori, the traditional healing practice of tangata whenua (the Indigenous peoples), as a separate entity from the context of CAM. Presently, there is no clear research in Aotearoa New Zealand on consumer use of CAM therapies for people who are healthy, unwell, have a chronic illness, or experience a hospital admission. However, discussions about which therapies and approaches are included when using the term CAM is ongoing. Anecdotally, health care consumers are exposed to a variety of CAM therapies, such as olive leaf oil, manuka honey, and arnica therapies (Andrews, Adams, Segrott \& Lui, 2012). Considering the variance in definitions and cultural perspectives of CAM, it is uncertain if nurses recognise the diverse layers and implications when using CAM as a generic term.

\section{Nurses' positioning}

When reviewing the literature on CAM in nursing, recurrent themes are evident. These include incentives and barriers to utilisation of CAM; the validity of the knowledge that nurses hold about CAM modalities and approaches; and the efficacy of CAM within health care (Lai, 2012; Rojas-Cooley \& Grant, 2009). Each theme provides an opportunity to further explore contextualising nursing and the value of CAM practice in New Zealand. Attempts to use CAM in primary health care helped to clarify the roles of health professionals, service providers, and CAM practitioners (Upsdell \& Jaye, 2011).

Many authors have highlighted various reasons for an increase in CAM approaches. These include patient dissatisfaction with mainstream health approaches; lack of patient self-efficacy; control over treatment choices; limited time for patient consultations in mainstream settings (Andrews et al., 2012; Hilbers \& Lewis, 2013); and dissatisfaction in one's workplace due to time constraints in providing holistic care (Kristiniak, 2011). Over a decade ago, several researchers explored how working within contrasting philosophical foundations of mainstream medicine and CAM can be a barrier to a more inquisitive discussion investigating connections between nursing as a caring profession and CAM (Andrews et al., 2012; Hall, Leach, Brosnan \& Collins, 2017). Exploring the characteristics of both, mainstream and CAM approaches, emphasises the dichotomy between the biomedical understandings of disease. For mainstream health approaches, the focus is on pathological processes, which is in contrast to CAM where the meaning of illness is a subjective experience of un-wellness. Gale (2014) has described these boundary experiences for practitioners when working across two paradigms as an active "process of construction, maintenance and communication of the dichotomy" (p. 809). This was confirmed by Orkaby and Greenberger (2015) who interviewed nurses working in secondary care who were using their knowledge of CAM. They found there was a clear coexistence of both a biomedical paradigm and holistic approach to nursing.

Further research to explore the attitudes nurses have towards CAM (Orkaby \& Greenberger, 2015; Rojas- 


\section{Nursing Praxis in New Zealand}

Cooley \& Grant, 2009) needs to be considered alongside the role-specific impact that the rise of CAM has had on the profession (Kristiniak, 2011; Lai, 2012) and how professional boundaries are challenged when working with CAM (Lai, 2012; Upsdell \& Jaye, 2011). Despite mission statements that outline and clarify the professional boundaries for nurses engaging with CAM therapies (College of Nurses Aotearoa, 2010; New Zealand Nurses Organisation [NZNO], 2011), CAM remains an important and a relevant part of contemporary health care provision (Shorofi \& Arbon, 2017).

The core nursing concepts of caring, empathy, humanitarianism, and patient-centred care are also in close alignment to the early contextualisation of CAM as established by the seminal researchers Micozzi (1996) and Zollman and Vickers (1999). Complementary and alternative medicine and nursing care focuses on building trusting, therapeutic relationships with health care consumers that places patient values at the centre of care delivery (Poreddi et al., 2016) where "nursing care and CAM share a holistic common ground" (Orkaby \& Greenberger, 2015, p. 20). However, demographical factors such as culture, knowledge, beliefs, values, and perceptions of nursing care will influence how CAM within an holistic approach to nursing is expressed, perceived, and evaluated (Kristiniak, 2011; NZNO, 2011).

\section{Holistic health care approach}

In Aotearoa New Zealand a holistic approach to nursing care includes the notion of culturally safe care, inclusiveness, and recognition of traditional Māori health care models and an increased awareness of other emergent traditional health frameworks (such as Traditional Chinese Medicine). Knowledge and research have established that cultural safety "becomes a point of conflict within an environment where discourse of traditional nursing care and medicine compete" (Richardson \& MacGibbon, 2010, p. 54). Acknowledging power differences in therapeutic relationships also means that the consumer holds a powerful position in this process, rather than being a passive recipient of therapy. The CAM paradigm advocates for inclusive processes and a focus on re-establishing equilibrium, which stands in contrast to the biomedical model that uses a reductionist approach which targets disease symptoms rather than fostering innate healing abilities (EUROCAM, 2014). However, valid questions for the nursing profession remain regardless of how this global rise in CAM therapies is rationalised.

Self-efficacy, consumer empowerment, validation, and alignment of CAM therapies with the fundamental nursing elements of holistic care are core beliefs in the CAM approach (Baer, 2016). While many research projects have focussed on the effects of ingested herbal, homeopathic remedies, and nutritional supplements (Choi et al., 2016), other projects have explored the use of touch (massage or other bodywork) and the essence of being with the patient as core elements of CAM (Kristiniak, 2011; Westman \& Blaisdell, 2016). Kristiniak (2011) supports this notion of being with and confirms this as part of the CAM paradigm noting that "complementary therapies add to the scope of nursing through interventions and holistic care that extend beyond the physical management of patients and their illness" (p. 6). Most CAM practitioners would place the restoration of balance and facilitation of innate healing abilities as the main target of their interventions (Poreddi et al., 2016). It is therefore not surprising that researchers have established a clear link between the use of CAM therapies and the nursing profession (Kristiniak, 2011).

\section{Translation into practice}

A limitation in establishing the link between CAM and holistic care is the lack of clarity around how nurses, working in mainstream areas, have acquired knowledge, experience, and utilisation of CAM (Chang \& Chang, 2015). If nurses practice CAM therapy, how are they acquiring and implementing this knowledge into clinical practice? Do nurses that use slippery elm or aloe for irritable bowel view this as CAM? While some studies 


\section{Nursing Praxis in New Zealand}

clearly point to a 'dual' professional practice with valid training completed in CAM therapies (Kristiniak, 2011), other studies highlight that knowledge is acquired via the internet or from personal experience (Shorofi \& Arbon, 2017). The projects that involve nurses in dual practitioner roles (MACCAH, 2004) could be reflective of the difficulties around managing professional practice situated within different healing paradigms (Kristiniak, 2011). The pool of literature in this context clearly shows the multifaceted influence of CAM and traditional healing on mainstream health practices (Hilbers \& Lewis, 2013; Mark \& Chamberlain, 2012).

It seems inevitable that nurses have to engage with CAM when caring for a wide range of consumers within various multi-cultural contexts. A deeper exploration of how CAM knowledge is acquired is required to inform nursing roles, practice, and positioning within the health environment in Aotearoa New Zealand. Acknowledging consumers' rights to CAM may be a motivator in the context of the importance of delivering culturally safe care. Baer (2009) describes that "New Zealand nurses have also become interested in incorporating CAM therapies into their treatment regimens..." (p. 140). However, there is a lack of knowledge about the importance CAM has on a nurse's professional identity nor how nurses consolidate health care knowledge from these different paradigms (Shorofi \& Arbon, 2017; Gale 2014).

\section{Conceptualising complementary and alternative medicine}

There is some literature that explores the link between nursing and CAM (Hall et al., 2017). There are limited studies exploring this connection in Aotearoa New Zealand, which tends to focus on how medical practitioners manage the demand and use of CAM by their patients in both primary health care (Poynton, Dowell, Dew \& Egan, 2006; Upsdell \& Jaye, 2011) and acute health care settings (Nicholson, 2006).
In Aotearoa New Zealand, research explored how nursing as a profession might be positioned in the context of CAM when faced by consumer demand or request for advice on CAM within the Plunket service (Aotearoa New Zealand's national well-child provider) (Lai, 2012). The study identified issues with structural and organisational barriers and ethical conflicts when integrating CAM into a mainstream setting. Questions raised profoundly challenged the role perceptions for nurses and the need for boundary clarification (Lai, 2012). All registered nurses practising in Aotearoa New Zealand are expected to have some knowledge on CAM therapies. In a position statement "Rongoā Māori and Complementary Therapies in Nursing Practice", NZNO (2011) stated:

\begin{abstract}
Regardless of whether a nurse chooses to utilise complementary therapy in practice or not, all nurses have a responsibility to be aware of the implications of complementary therapy for those people who choose to utilise these as part of their health care. This may include providing resource material, education, and/or referrals to qualified practitioners. (p. 4).
\end{abstract}

Postmodern views and perspectives have influenced an increasingly independent consumer group leading to the development of more balanced therapeutic relationships between health care providers and the recipients of care (Gale, 2014). In addition, the principles of culturally safe care require the appreciation of different values and beliefs, placing the consumer, rather than the nurse, at the centre of care (Richardson \& MacGibbon, 2010). The positioning of nurses who are practising CAM therapies alongside their mainstream roles, together with the link between cultural safety and CAM needs further exploration to be contextually situated within nursing in New Zealand (Lai, 2012).

\section{Implications}

The professional nursing bodies in Aotearoa New Zealand have safety and management guidelines for 


\section{Nursing Praxis in New Zealand}

CAM (College of Nurses Aotearoa, 2010; NZNO, 2011). Complementary and alternative medicine can be used as long as enrolled nurses, registered nurses, and nurse practitioners work within their regulated scope of practice and competencies (Nursing Council of New Zealand, 2016). What is arguably of value to future nursing practice in Aotearoa New Zealand, is the effect and impact the use and knowledge of CAM approaches and therapies might have on care delivery for consumers and their whānau (family, extended family). Further, undertaking such extended practice might also have positive impacts on nurses' professional identity. Recognising that a juxtaposition exists between the biomedical discourse inherent in Western health systems and nursing practice, by embracing the knowledge and paradigms of CAM potentially can add value to the nursing profession. Health care providers are caring for an increasing ethnically diverse patient group (Statistics New Zealand, 2013), which requires nurses to not only apply principles of cultural safety confidently and competently but also requires an ability to acknowledge a variety of healing approaches based on different worldviews (Richardson \& MacGibbon, 2010).

Incorporating collaborative care and pluralistic views of what health means to individuals and whānau can be challenging when providing care exclusively within a biomedical, Western paradigm (Orkaby \& Greenberger, 2015). Nurses are affected by these changes in consumer demand and are increasingly required to comment on the use of CAM modalities and therapies (Hall et al., 2017; Kristiniak, 2011). Nurses using a CAM therapy alongside their mainstream knowledge might face a variety of challenges not yet investigated within the Aotearoa New Zealand context (Lai, 2012). These varied contexts of CAM interactions with mainstream therapies leave space for a wide range of considerations. Questions pertaining to nursing philosophy, cultural safety, role behaviour, and inter-professional collaboration still need to be addressed and opportunities created that allow for discussions about the possible barriers or facilitators for the use of CAM. Richardson and MacGibbon (2010) examined the positioning of nurses who cared for someone with a different view than their own and what this triggered for the nurse: "We tend to look at patients who won't do what we want them to do and think "What is wrong with them?" (p. 54). This statement highlights how others' views are dismissed as contrary to accepted knowledge. Richardson and MacGibbon referred to othering as views not inherent within the dominant discourse. Further, the statement represented a historic incumbent notion of power and knowledge linked to the biomedical approach to health care (Gale, 2014). Future research projects need to explore how nurses use CAM knowledge and how this knowledge affects nursing assessment skills and engagement with care of patients, scope of practice, role, professional identity, philosophical positioning, relationships with colleagues, and liaison with other services, within the context of providing culturally safe care within Aotearoa New Zealand.

\section{Conclusion}

This discussion paper aimed to pose potential questions arising for nurses in Aotearoa New Zealand when engaging with or utilising their theoretical and practical knowledge of CAM whilst working in a mainstream setting. Despite the increased use of CAM therapies in the past 20 years, there is a gap in the literature on how paradigmatic alignments or discrepancies between nursing and CAM might influence care delivery and professional attitudes. Globally, studies have reported that the core tenets of nursing practice are aligned to the intrinsic features of the CAM approach to health and well-being, but these findings need to be validated for nursing within Aotearoa New Zealand. Further research and insight into whether nurses practising CAM are affected by a variety of constraints and how their practice might be influenced when working across different paradigmatic boundaries is required. To better position nurses within this increasingly diverse health environment of consumers' therapeutic choices, nurses need to explore these questions, so 


\section{Nursing Praxis in New Zealand}

our roles as health care providers are progressively validated and acknowledged. Initiating discussions between various CAM practitioners, traditional healers, and nurses, and inviting nurses practising within both paradigms to share their CAM experiences, will facilitate a better understanding of practice within pluralistic healing paradigms. Increased knowledge and use of CAM therapies within Aotearoa New Zealand will facilitate a better understanding of consumer driven choices in care delivery options, promoting culturally safe practice. Creating a platform for these discussions will enhance inter-professional collaboration and foster liaison with various healers and practitioners of traditional medicine, such as rongoā Māori, and other healing modalities increasingly common within Aotearoa New Zealand's multicultural population.

\section{References}

Ahuriri-Driscoll, A., Baker, V., Hepi, M., Hudson, M., Mika, C., \& Tiakiwai, S. (2008). The future of rongoā Maori: Wellbeing and sustainability. Wellington, New Zealand: Institute of Environmental Science and Research Ltd \& Ministry of Health.

Andrews, G. J., Adams, J., Segrott, J., \& Lui, C. W. (2012). The profile of complementary and alternative medicine users and reasons for complementary and alternative medicine use. In J. J. Adam, G. J. Andrews, J. Barnes, A. Broom, \& P. Magin (Eds.), Traditional, complementary and integrative medicine. An international reader (pp.11-17). Basingstoke, UK: MacMillan Education.

Baer, H. (2016). Complementary medicine in Australia and New Zealand: Its popularisation, legitimation, and dilemmas (2 ${ }^{\text {nd }}$ ed.). New York, NY: Routledge.

Chang, H., \& Chang, H. (2015). A review of nurses' knowledge, attitudes, and ability to communicate the risks and benefits of complementary and alternative medicine. Journal of Clinical Nursing, 24(11-12), 1466-1466. doi: 10.1111/jocn.12790

Choi, J., Eom, S., Kim, J., Kim, S., Huh, E., \& Kim, H., ... Oh, M. (2016). A comprehensive review of recent studies on herb-drug interaction: A focus on pharmacodynamic interaction. Journal of Alternative and Complementary Medicine, 22(4), 262-79. doi: 10.1089/acm.2015.0235

College of Nurses Aotearoa New Zealand. (2010). The professional indemnity insurance programme. Retrieved from https://www. nurse.org.nz/indemnity-insurance-cover.html

Eardley, S., Bishop, F., Prescott, P., Cardini, F., Brinkhaus, B., Santos-Rey, K., ... Lewith, G. (2010). CAM Use in Europe - The patients' Perspective. Part I: A systematic literature review of CAM prevalence in the EU. Final Report of CAMbrella, Munich, Germany. Retrieved from http://camdoc.eu/Pdf/CAMbrella-WP4-part_1final.pdf

EUROCAM. (2014). CAM 2020: The contribution of complementary and alternative medicine to sustainable healthcare in Europe. Retrieved from http://ehtpa.eu/pdf/CAM2020-FINAL.pdf

European Federation of Complementary and Alternative Medicine. CAM status in Europe. Retrieved from http://www.efcam.eu/ cam/cam-status-in-europe/

Frass, M., Strassl, R., Friehs, H., Müllner, M., Kundi, M., \& Kaye, A. (2012). Use and acceptance of complementary and alternative medicine among the general population and medical personnel: A systematic review. The Ochsner Journal, 12(1), 45-56.

Gale, N. (2014). The sociology of traditional, complementary and alternative medicine. Sociology Compass, 8(6), 805-822. doi: $10.1111 /$ soc4.12182

Hall, H., Leach, M., Brosnan, C., \& Collins, M. (2017). Nurses' attitudes towards complementary therapies: A systematic review and meta-synthesis. International Journal of Nursing Studies, 69(April), 47-56. doi: 10.1016/j.jjnurstu.2017.01.008

Hilbers, J., \& Lewis, C. (2013). Complementary health therapies: Moving towards an integrated health model. Collegian, 20(1), 51-60. doi: 10.1016/j.colegn.2012.03.004

Kristiniak, S. B. (2011). Exploring the experiences of complementary nurses: A qualitative phenomenological study. (Doctoral dissertation, University of Phoenix, Phoenix, Arizona, US.) Retrieved from https://search.proquest.com/openview/5607ba9f860923511d7e2 ca4998e 55c2/1?pq-origsite $=$ gscholar $\&$ cbl $=18750 \&$ diss $=y$

Lai, S. L. H. (2012). Perceptions and attitudes of New Zealand Plunket nurses toward the use of complementary and alternative medicine in children. (Master's dissertation, Unitec Institute of Technology, Auckland, New Zealand.) Retrieved from https://hdl.handle. net/10652/2365

Mark, G., \& Chamberlain, K. (2012). Māori healers' perspectives on cooperation with biomedicine. The Australian Community Psychologist, 24(1), 97-105. 


\section{Nursing Praxis in New Zealand}

Micozzi, M. (Ed.). (2014). Fundamentals of complementary and alternative medicine. (5 $5^{\text {th }}$ ed.). St. Louis, MO: Elsevier/Saunders.

Ministerial Advisory Committee on Complementary and Alternative Health. (2002). Terminology in complementary and alternative health. Retrieved from http://www.moh.govt.nz/notebook/nbbooks.nsf/0/EDF8E1185848CE10CC256D1C00768E32/\$file/ Terminology-in-complementary-and-alternative-health.pdf

Ministerial Advisory Committee on Complementary and Alternative Health. (2004). Complementary and alternative health care in New Zealand: Advice to the Minister of Health. Wellington, New Zealand: Ministry of Health. Retrieved from https://www.health. govt.nz/publication/complementary-and-alternative-health-care-new-zealand-0

Ministry of Health. (2008). A portrait of health. Key results of the 2006/07 New Zealand health survey. Retrieved from http://www. moh.govt.nz/NoteBook/nbbooks.nsf/0/9FF50018D90C43D3CC2574640009AE76/\$file/portrait-of-health-june08.pdf

National Center for Complementary and Integrative Health. (2016a). Complementary, alternative, or integrative health: What's in a name? Retrieved from https://nccih.nih.gov/health/integrative-health

National Center for Complementary and Integrative Health. (2016b). Safe use of complementary health products and practices. Retrieved from https://nccih.nih.gov/health/safety

New Zealand Nurses Organisation. (2011). Rongoā Māori and complementary therapies in nursing practice. Retrieved from https:// www.nzno.org.nz/Portals/0/publications/Rongo\%C4\%81\%20M\%C4\%81ori\%20and\%20Complementary\%20Therapies\%20in\%20 Nursing\%20Practice.pdf

Nicholson, T. (2006). Complementary and alternative medicine (including traditional Māori treatments) used by presenters to an emergency department in New Zealand: A survey of prevalence and toxicity. The New Zealand Medical Journal, 119(1233), 9-21.

Nursing Council of New Zealand. (2016). Nurse competencies for registered nurses. Wellington, New Zealand: Nursing Council of New Zealand. Retrieved from http://www.nursingcouncil.org.nz/Nurses

O'Connor, B. B., Calabrese, C., Cardeña, E., Eisenberg, D. M., Fincher, J., Hufford, D. J., ... Zhang, X. (1997). Defining and describing complementary and alternative medicine. Alternative Therapies in Health and Medicine, 3(2), 49-57.

Orkaby, B., \& Greenberger, C. (2015). Israeli nurses' attitudes to the holistic approach to health and their use of complementary and alternative therapies. Journal of Holistic Nursing, 33(1), 19-26. doi: 10.1177/0898010114542876

Poreddi, V., Thiyagarajan, S., Swamy, P., Ramachandra; Gandhi, S., Thimmaiah, R., \& Badamath, S. (2016). Nursing students attitudes and understanding of complementary and alternative therapies: An Indian perspective. Nursing Education Perspectives, 37(1), 32-37. doi: $10.5480 / 14-1319$

Poynton, L., Dowell, A., Dew, K., \& Egan, T. (2006). General practitioners' attitudes toward (and use of) complementary and alternative medicine: A New Zealand nationwide survey. The New Zealand Medical Journal, 119(1247), 2361.

Richardson, F., \& MacGibbon, L. (2010). Cultural safety: Nurses' accounts of negotiating the order of things. Women's Studies Journal, 24(2), 54-65.

Rojas-Cooley, M., \& Grant, M. (2009). Complementary and alternative medicine: Oncology nurses' knowledge and attitudes. Oncology Nursing Forum, 36, 217-224. doi: 10.1188/09.ONF.217-224

Shorofi, S., \& Arbon, P. (2017). Complementary and alternative medicine (CAM) among Australian hospital-based nurses: Knowledge, attitude, personal and professional use, reasons for use, CAM referrals, and socio-demographic predictors of CAM users. Complementary Therapies in Clinical Practice, 27, 37-45. doi: 10.1016/j.ctcp.2017.03.001

Statistics New Zealand. (2014). 2013 Census QuickStats about culture and identity. Retrieved 2019 from https://www.nbr.co.nz/sites/ default/files/quickstats-culture-identity.pdf

Tabish, S. A. (2008). Complementary and alternative health care: Is it evidence-based? International Journal of Health Sciences, 2(1), V-IX.

Upsdell, M., \& Jaye, C. (2011). Engaging with complementary and alternative medicine in general practice. Journal of Primary Health Care, 3(1), 29-34. doi: 10.1071/HC11029

Westman K. F., \& Blaisdell, C. (2016). Many benefits, little risk: The use of massage in nursing practice. American Journal of Nursing, 116(1), 34-39. doi: 10.1097/01.NAJ.0000476164.97929.f2

World Health Organization. (2013). WHO traditional medicine strategy 2014-2023. Geneva, Switzerland: World Health Organization. Retrieved from http://apps.who.int/medicinedocs/documents/s21201en/s21201en.pdf

Zollman, C., \& Vickers, A. (1999). What is complementary medicine? British Medical Journal, 319(7211), 693 -696. doi: 10.1136/ bmj.319.7211.693 